\title{
BMJ Open Post-treatment surveillance testing of patients with colorectal cancer and the association with survival: protocol for a retrospective cohort study of the Surveillance, Epidemiology, and End Results (SEER)-Medicare database
}

Robert B Hines, ${ }^{1}$ Md Jibanul Haque Jiban, ${ }^{2}$ Kanak Choudhury, ${ }^{2}$ Victoria Loerzel, ${ }^{3}$ Adrian V Specogna, ${ }^{4}$ Steven P Troy, ${ }^{5}$ Shunpu Zhang ${ }^{2}$

To cite: Hines RB, Jiban MJH, Choudhury K, et al. Posttreatment surveillance testing of patients with colorectal cancer and the association with survival: protocol for a retrospective cohort study of the Surveillance, Epidemiology, and End Results (SEER)Medicare database. BMJ Open 2018;8:e022393. doi:10.1136/ bmjopen-2018-022393

- Prepublication history for this paper is available online. To view these files, please visit the journal online (http://dx.doi. org/10.1136/bmjopen-2017022393).

Received 14 February 2018 Revised 2 April 2018 Accepted 4 April 2018

Check for updates

${ }^{1}$ Internal medicine, University of Central Florida College of Medicine, Orlando, Florida, USA ${ }^{2}$ Statistics, University of Central Florida College of Sciences, Orlando, Florida, USA

${ }^{3}$ University of Central Florida College of Nursing, Orlando, Florida, USA

${ }^{4}$ University of Central Florida College of Health and Public Affairs, Orlando, Florida, USA

${ }^{5}$ University of Central Florida College of Medicine, Orlando, Florida, USA

Correspondence to

Dr Robert B Hines;

robert.hines@ucf.edu

\section{ABSTRACT}

Introduction Although the colorectal cancer (CRC) mortality rate has significantly improved over the past several decades, many patients will have a recurrence following curative treatment. Despite this high risk of recurrence, adherence to CRC surveillance testing guidelines is poor which increases cancer-related morbidity and potentially, mortality. Several randomised controlled trials (RCTs) with varying surveillance strategies have yielded conflicting evidence regarding the survival benefit associated with surveillance testing. However, due to differences in study protocols and limitations of sample size and length of follow-up, the RCT may not be the best study design to evaluate this relationship. An observational comparative effectiveness research study can overcome the sample size/ follow-up limitations of RCT designs while assessing realworld variability in receipt of surveillance testing to provide much needed evidence on this important clinical issue. The gap in knowledge that this study will address concerns whether adherence to National Comprehensive Cancer Network CRC surveillance guidelines improves survival. Methods and analysis Patients with colon and rectal cancer aged 66-84 years, who have been diagnosed between 2002 and 2008 and have been included in the Surveillance, Epidemiology, and End Results-Medicare database, are eligible for this retrospective cohort study. To minimise bias, patients had to survive at least 12 months following the completion of treatment. Adherence to surveillance testing up to 5 years post-treatment will be assessed in each year of follow-up and overall. Binomial regression will be used to assess the association between patients' characteristics and adherence. Survival analysis will be conducted to assess the association between adherence and 5 -year survival.

Ethics and dissemination This study was approved by the National Cancer Institute and the Institutional Review Board of the University of Central Florida. The results of this study will be disseminated by publishing in the peerreviewed scientific literature, presentation at national/ international scientific conferences and posting through social media.
Strengths and limitations of this study

The use of numerous Medicare claims datasets results in redundancy of information to ensure accuracy of exposure (surveillance testing) information.

- The use of the Surveillance, Epidemiology, and End Results-Medicare database which contains a large number of colorectal cancer cases with extensive follow-up information, overcoming the sample size and power limitations of previously conducted randomised controlled trials.

- The use of a representative US cancer patient population which will allow an examination of the effectiveness of surveillance testing reflecting the wide range of surveillance testing received in real-world clinical settings.

- This is an observational study and thus, the lack of patient randomisation according to receipt of surveillance testing could introduce bias by unmeasured confounding.

- The use of an older, Medicare population may not be generalisable to younger patients with private health insurance.

\section{INTRODUCTION}

Approximately 9\% ( $\mathrm{n}=1452$ 040) of the estimated 15.5 million cancer survivors in the USA are patients surviving colorectal cancer (CRC). ${ }^{1}$ Although the CRC mortality rate has substantially improved over the past several decades, ${ }^{2}$ tumour recurrence after curative treatment is high. To wit, up to $13 \%$ of stage $\mathrm{I}^{3}$ and approximately $40 \%$ of stage $\mathrm{II} / \mathrm{III}^{4} 5$ patients will experience a recurrence. The primary goal of CRC surveillance testing is to improve survival "with the hope of providing a better outcome than if recurrence had been detected later as a result of symptoms'. ${ }^{6}$ According to the National 
Comprehensive Cancer Network (NCCN), surveillance testing in CRC survivors consists of: physical examinations, carcinoembryonic antigen (CEA) blood tests, CT scans and colonoscopy. ${ }^{78}$ Despite high consistency in testing recommendations between guideline issuing groups, ${ }^{7-10}$ adherence is poor ${ }^{11-14}$ which increases morbidity and potentially, mortality. ${ }^{14-18}$ Thus, efforts to increase adherence to surveillance guidelines appear long overdue. However, some clinicians have questioned the evidence base supporting surveillance recommendations. In 2007, two independent meta-analyses of randomised controlled trials (RCTs), which included nearly identical studies, concluded that more intensive surveillance improves overall survival (OS), but better cancer-specific survival (CSS) was not demonstrated. ${ }^{16}{ }^{18}$ However, only two older, smaller RCTs evaluated this outcome. ${ }^{417}$

Since the publication of the two aforementioned meta-analyses in $2007,{ }^{16} 18$ two additional meta-analyses were published in 2016, one by the same authors as the previously published report. ${ }^{1920}$ Both of these more recent meta-analyses concluded that more intensive surveillance in patients with CRC does not improve survival (neither OS nor CSS). As noted, this conclusion by Jeffery et $a l^{19}$ was a reversal of their earlier position on CRC and OS. ${ }^{16}$ One limitation of the meta-analyses that have been published on the topic concerns the heterogeneous surveillance strategies adopted by the included RCTs, which makes combining study results problematic. For example, in the RCT by Wang $e t$ al, ${ }^{21}$ Patients with Dukes' stages I-III CRC were followed after radical surgery. Both groups received intensive surveillance consisting of clinical examination, CEA testing, chest X-ray and liver imaging (via CT or ultrasound). In the control group, patients received colonoscopy at 6 (if not done preoperatively), 30 and 60 months whereas in the intervention group, patients received colonoscopy at every visit. Colonoscopy can only detect intraluminal recurrence or metachronous tumours with no ability to detect metastatic disease. ${ }^{14}$ Thus, although there appeared to be some benefit of more frequent colonoscopic testing (higher detection of asymptomatic cancers and reoperation with curative intent) there was no significant OS benefit. In the Gruppo Italiano Lavoro per la Diagnosi Anticipata (GILDA) study, Rosati $e t a l^{22}$ failed to detect a survival advantage for more 'intensive' surveillance in patients with CRC. As we pointed out in a letter to the editor, ${ }^{23}$ patients in the control arm of this study actually received intensive surveillance testing and patients in the intervention arm received additional testing beyond that recommended by guidelines which could be described as overtreatment. As a final example of problematic inclusion of heterogeneous RCTs in the aforementioned meta-analyses, there were two studies ${ }^{24} 25$ in which both control and intervention groups received identical surveillance testing schedules, but the studies compared delivery by type of provider: either surgeon versus nurse ${ }^{24}$ or surgeon versus general practitioner. ${ }^{25}$ There was no survival difference in either RCT although in the meta-analyses, surgeon follow-up was considered part of the 'intensive' surveillance group. These examples highlight problems concerning the comparability of RCTs combined in meta-analyses which limits the ability to draw broad conclusions concerning the potential survival benefit of post-treatment surveillance testing.

The highest quality RCT on the issue of surveillance testing was conducted by Primrose $e t a l^{15}$ in the Follow-Up After Colorectal Surgery RCT, conducted in the UK. This study revealed that surveillance with CEA/CT increased the likelihood of curative resection by three times compared with standard follow-up care, but no differences in survival were indicated. ${ }^{15}$ There are at least two potential reasons for these null results. One, clinicians of patients in the minimal follow-up group could request CT scan of the chest, abdomen and pelvis at 12-18 months which can detect locoregional and metastatic recurrence. Nine out of 37 patients with recurrence in the minimum follow-up group had scheduled CT testing which positively identified the recurrence. A second reason for the lack of a survival difference in this trial is due to improvements in the treatment of primary and recurrent CRC due to surgical advances, improved chemotherapeutics and combined therapy with biologics. The implication of this is that patients would have to be followed for an extensive length of time in order for enough deaths to occur to detect a survival benefit, a limitation that Primrose et $a l^{15}$ acknowledged. This also relates to a statistical power issue of RCT designs in general, whereby it is usually cost prohibitive to enrol enough participants and follow them for a sufficient length of time to observe sometimes clinically meaningful, but relatively small survival benefits that reach statistical significance. It should be noted that the ongoing, multicentre Assessment of Frequency of Surveillance after Curative Resection in Stage II and III Colorectal Cancer (COLOFOL) trial aims to overcome the statistical power issue of previous RCTs. This trial is the largest of any RCT conducted on the issue of CRC surveillance having enrolled 2509 participants. ${ }^{26}$ While not without their own limitations, observational epidemiological studies can overcome statistical power issues while providing evidence on the comparative effectiveness of surveillance testing reflecting what patients actually receive in real-world clinical settings.

Due to the limitations of the RCTs described above and the lack of definitive evidence to inform clinical guidelines on this issue, there is currently debate regarding the optimal frequency of surveillance testing and even if it should be done at all. ${ }^{19}$ Observational comparative effectiveness research (CER) using the Surveillance, Epidemiology, and End Results database combined with Medicare claims (SEER-Medicare) is ideal to provide evidence to physicians on this highly important clinical issue while overcoming the limitations of RCT designs. ${ }^{27}{ }^{28}$ In this protocol manuscript, we describe our ongoing retrospective, comparative effectiveness cohort study of the SEER-Medicare database. The gap in knowledge that this study will address concerns whether adherence to NCCN CRC surveillance guidelines ${ }^{78}$ improves survival 
for a broad population of older patients with CRC. We will assess whether the frequency of testing in the current guidelines is the optimal strategy or if less/more testing achieves worse, equivalent or better outcomes. We will also evaluate specific test components which make up the guidelines and the association with CSS and OS. Secondarily, we will assess the association of sociodemographic and clinical characteristics of CRC survivors with the likelihood of receiving surveillance testing that is adherent with guidelines.

\section{METHODS AND ANALYSIS}

\section{Study design and population}

A retrospective cohort design will be used for this CER study. Cases of colon and rectal cancer diagnosed between 2002 and 2008 and reported to the SEER-Medicare database are eligible. We have the following inclusion criteria:

1. CRC cases must have the following International Classification of Diseases for Oncology Edition 3 $(\text { ICD-O-3 })^{29}$ topographical codes corresponding to their diagnosis: C18.0, C18.2, C18.3, C18.4, C18.5, C18.6, C18.7, C18.8, C18.9, C20.9.

2. Cases must be classified within disease stages I-III.

3. Cases must be between 66 and 84 years of age at diagnosis.

4. Cases must be continuously enrolled in Medicare parts A and B from 13 months before diagnosis to at least 12 months following treatment completion.

Cases will be excluded from the study if:

1. They had a previous cancer diagnosis.

2. They had more than one primary tumour at diagnosis.

3. They were diagnosed with a second primary tumour before completing treatment.

4. Tumour histology is other than adenocarcinoma.

5. They had managed care coverage at any time during the follow-up period.

6. There is no evidence that they received surgery as treatment for their cancer.

7. They received treatment outside of standard time frames.

8. They died within 1 year following the completion of treatment.

The rationale for excluding patients who died within 1 year of treatment completion is to eliminate the potential for immortal time bias. ${ }^{30}$ In order to be exposed to surveillance testing, patients had to survive for a certain period of time to undergo testing and live long enough following the completion of treatment for surveillance testing to be considered part of the causal pathway in survival. For example, it could be argued that patients who died of cancer within 1 year of completing treatment were never disease free and therefore, any surveillance testing that occurred would not be predictive of CSS. Surveillance testing is appropriate only if the patient underwent curative treatment with no evidence of disease at treatment completion.

\section{Patient and public involvement}

This study is informed by the literature concerning patients with CRC likelihood of receiving surveillance testing that is adherent to treatment guidelines, ${ }^{11-14} 31-33$ predictors of receiving surveillance tests ${ }^{33-35}$ and previous studies addressing the relationship of surveillance testing with survival. ${ }^{4-22} 25$ As this is a retrospective cohort study of SEER-Medicare data, the patients included in this study were not involved in the study design nor were they recruited. Furthermore, as we do not have individually identifiable information on CRC cases included in this study, study participants will not be notified of the study's results.

\section{Disease stage}

CRC cases will be classified into disease stage groups according to the Eighth Edition of the American Joint Committee on Cancer tumour, node, metastasis classification system. ${ }^{36}$ The degree of detail in this staging system is not always available in the SEER-Medicare database, particularly for patients diagnosed in the earlier years of the study. We developed an algorithm to categorise each patient into the appropriate disease stage based on a number of variables. These variables included previous versions of the AJCC stage groupings, $\mathrm{T}$ and $\mathrm{N}$ stage definitions, and variables documenting the extent of disease. Although the variable names differed for these two time periods, the same process was used for patients diagnosed in 2002-2003 and for the years 2004-2008 with the latter period corresponding to the collaborative staging system currently in use. It is important to note that the degree of detail corresponding to disease stage (eg, IIIA, IIIB) is not required as surveillance guidelines follow broad stage groupings (eg, I, II, III). However, having more detailed stage information will allow us to examine heterogeneity of effect estimates within stage.

\section{Treatment status}

In order to minimise potential sources of bias, we must correctly classify receipt of treatment within accepted standards of time. This is necessary to limit variability in the amount of time from diagnosis to completion of treatment. As patients are required to live 1 year following the completion of treatment, it is necessary to reduce variability in this time period for reasons of immortal time bias as described above. This will be achieved by requiring that CRC cases who received a particular therapy do so within accepted time frames. For example, for patients with colon cancer, as defined in other studies, ${ }^{37} 38$ receipt of surgery $\leq 90$ days of diagnosis will be considered as receiving cancer-directed surgery (required for study inclusion). Receipt of chemotherapy will be recorded as positive if it occurred within 120 days of the surgery date. Patients who received chemotherapy $>120$ days following surgery but $\leq 1$ year of diagnosis date will be excluded. Receipt of chemotherapy $>1$ year following diagnosis will be considered as treatment for disease progression/recurrence and will not be used to define the final treatment date. 
For patients with rectal cancer, those who received neoadjuvant radiation ( \pm chemotherapy) $\leq 90$ days following diagnosis will be considered as having received this therapy. As there is evidence that a longer interval between neoadjuvant radiation and surgery may promote more complete pathological response, ${ }^{39}$ patients who receive surgery $\leq 150$ days following the completion of radiotherapy will be retained in the study. Last, patients will be considered as having received adjuvant chemotherapy if they do so within 120 days of surgery.

Based on our previous studies, ${ }^{40}{ }^{41}$ the proportion of patients with CRC who received a particular therapy outside of the recommended time frames described above is expected to be very small.

\section{SEER-Medicare data files}

The following files from the National Cancer Institute's SEER-Medicare linked database will be used to conduct this study: Patient Entitlement and Diagnosis Summary Files (PEDSF), Medicare Provider Analysis and Review (MEDPAR), carrier claims, outpatient claims, Durable Medical Equipment (DME) and hospice. There are redundancies in the type of information that can be obtained from each file type, and this will be leveraged to ensure completeness/accuracy of data.

As noted in the PEDSF documentation, 'The PEDSF file contains one record per person for individuals in the SEER Program data base who have been matched with Medicare enrolment records. ${ }^{42}$ This file contains information on Medicare eligibility/enrolment and demographic, cancer-related, and treatment-related (surgery, radiation) information. This file will be considered the 'master' file on which relevant information from the other file types will be added. The MEDPAR file contains claims information resulting from inpatient admissions to hospitals and skilled nursing facilities. This information will be used to obtain data on type of treatment (eg, surgery) and relevant surveillance information (eg, colonoscopy). Treatment-related and post-treatment surveillance testing information will be obtained from the carrier claims file which includes claims from a variety of provider types and specialties. Claims from the outpatient file include billed procedures from outpatient providers including institutional and hospital-based outpatient departments. Similar to the carrier claims files, treatment and surveillance testing information will be obtained from this data file. Claims information from the DME file include diagnoses, procedures and oral equivalents of intravenous chemotherapy. As patients who begin receiving hospice care are terminal, it is no longer appropriate for them to undergo surveillance testing. It is important to identify these patients (via the hospice file) and classify their surveillance experience prior to receiving hospice care. Information on other diagnoses in the 12 months preceding the cancer diagnosis will be used for comorbidity assessment. ${ }^{43}$ This information will be obtained from MEDPAR, carrier claims and outpatient files.
Obtaining specific dates for diagnosis and procedures related to treatment and surveillance testing is an important component in this study. For example, although the PEDSF file contains the month/year of diagnosis, the day is missing. In order to obtain diagnosis date, we will select the earliest date corresponding to CRC diagnosis codes in the MEDPAR, carrier claims and outpatient files. If this date matches the year/month designated in PEDSF, this date will be designated as the diagnosis date. If the month/year of the earliest date corresponding to CRC diagnosis from the aforementioned data files does not match the month/year of diagnosis in PEDSF, the diagnosis date will be considered as the 15th of month of the given month/year diagnosis in PEDSF. In addition, obtaining the final date of treatment is necessary as this is the time point from which patients are required to live 1 year for study eligibility. This information can be obtained from the files above by searching the claims data for treatment procedures corresponding to ICD-9 (International Classification of Diseases, Ninth Revision) diagnosis codes for CRC (153.0, 153.1, 153.2, 153.3, 153.4, 153.7, 153.8, 153.9, 154.1). In this manner, relevant cancer-related procedures with dates can be obtained from the claims data.

There are a number of authors which have provided comprehensive lists of ICD-9, Current Procedural Terminology (CPT), Healthcare Common Procedure Coding System (HCPCS) diagnostic/procedure codes for CRC diagnosis and treatment ${ }^{44}$ as well as chemotherapy/radiotherapy codes for all cancers. ${ }^{45}$ The relevant codes that will be used to capture surveillance information are: physical examination (CPT: 99201-99 205, 99211-99 215, 99241-99 245), CEA (CPT: 82378), colonoscopy (ICD-9: 45.23, 45.25; CPT: 44388-44 394, 44 397, 45 355, 45 378-45 393, 45 398; HCPCS: G0105, G0121) and CT scans (ICD-9: 87.41, 88.01, 88.38; CPT: 71 250, 71 260, 71 270, 71 275, 74 150, 74 160, 74 170, 74175-74 178, $72191-72$ 194).

\section{Exposure assessment: adherence to NCCN surveillance guidelines}

Current NCGN surveillance guidelines for stages I-III patients with CRC are shown in table 1 . There are some additional nuances to the guidelines; table 1 is a simplified version. For example, if advanced adenoma (villous polyp, polyp $>1 \mathrm{~cm}$ or high-grade dysplasia) is detected on colonoscopy, repeat colonoscopy is recommend in the next year. However, as we will not have the ability to detect the presence of advanced adenoma via claims data, 1-year repeat colonoscopy will not be considered in determining guideline adherence. For CRC survivors in our study, patients who meet the minimum testing schedule in each year following the completion of treatment will be considered adherent.

The assessment of adherence to surveillance guidelines begins from the last date of treatment (ie, the date of surgery or the end of adjuvant chemotherapy treatment) until: (1) the patient dies of a non-cancer-related death, 


\begin{tabular}{|c|c|}
\hline \multicolumn{2}{|l|}{ Colon cancer } \\
\hline Stage I & $\begin{array}{l}\text { Colonoscopy at } 1 \text { year with repeat } \\
\text { colonoscopy at } 3 \text { years and every } 5 \text { years } \\
\text { thereafter. }\end{array}$ \\
\hline Stages II/III & $\begin{array}{l}\text { History and physical examination } \\
\text { every } 3-6 \text { months for } 2 \text { years and every } \\
6 \text { months for a total of } 5 \text { years. CEA } \\
\text { testing is recommended at baseline and } \\
\text { corresponding to the frequency of history } \\
\text { and physical examination. Colonoscopy } \\
\text { is recommended approximately } 1 \text { year } \\
\text { after resection, at } 3 \text { years and every } \\
5 \text { years thereafter. Chest, abdominal and } \\
\text { pelvic CT scans are recommended every } \\
6-12 \text { months up to } 5 \text { years. }\end{array}$ \\
\hline \multicolumn{2}{|l|}{ Rectal cancer } \\
\hline Stage I & $\begin{array}{l}\text { Colonoscopy at } 1 \text { year with repeat } \\
\text { colonoscopy at } 3 \text { years and every } 5 \text { years } \\
\text { thereafter. }\end{array}$ \\
\hline Stages II/III & $\begin{array}{l}\text { History and physical examination } \\
\text { every } 3-6 \text { months for } 2 \text { years and every } \\
6 \text { months for a total of } 5 \text { years. CEA } \\
\text { testing is recommended at baseline and } \\
\text { corresponding to the frequency of history } \\
\text { and physical examination. Colonoscopy } \\
\text { is recommended approximately } 1 \text { year } \\
\text { after resection, at } 3 \text { years and every } \\
5 \text { years thereafter. Chest, abdominal and } \\
\text { pelvic CT scans are recommended every } \\
6-12 \text { months for up to } 5 \text { years. }\end{array}$ \\
\hline
\end{tabular}

CEA, carcinoembryonic antigen; NCCN, National Comprehensive Cancer Network.

(2) 6 months prior to a cancer-related death, (3) the patient begins receiving hospice treatment, (4) the last date of continuous enrolment in Medicare parts A and B or (5) 5 years follow-up from final treatment date. We are excluding the 6 months before a patient's cancer-related death because it is likely that the clinical team will know the cancer has returned and surveillance will no longer be appropriate.

To obtain a nuanced understanding of the relationship between adherence and survival, we will classify CRC survivors according to adherence status in a number of ways. Consistent with NCCN surveillance guidelines, each CRC survivor will be categorised according to adherence status in each year of survival following the completion of treatment. Thus, for each year of follow-up, we will expand on the classification adopted by Mollica et $a l^{33}$ and classify participants as adherent, mostly adherent (non-adherent for one recommended surveillance test), partially adherent (non-adherent for two tests) and poorly adherent (non-adherent for $\geq 3$ tests) in each year of follow-up. Based on their yearly classification, patients will be given an overall designation as adherent/mostly adherent (either adherent/mostly adherent for all years of follow-up), partially adherent (contains 1 or more years designated as partially adherent) and poorly adherent (contains 1 or more years designated as poorly adherent). This classification will allow us to test the relationship between the frequency of surveillance testing and survival. Furthermore, we will use a similar methodology to classify study participants as more versus less adherent for specific tests (eg, CEA, CT) in the overall follow-up period. The above description applies to patients with stages II/III CRC who are required to undergo multiple surveillance tests each year. For stage I patients, a similar methodology will be used such that patients are classified according to adherence status based on the timing of colonoscopy following cancer-directed surgery: year 1 (adherent), year 2 (mostly adherent), year 3 (partially adherent), no colonoscopy in years 1-3 (poorly adherent).

\section{Primary and secondary exposure-outcome relationships}

Our primary exposure-outcome relationship is to assess the association between levels of surveillance testing (adherent/mostly adherent vs partially and poorly adherent) with both CSS and OS. Our secondary exposure-outcome relationship is to assess the association between patients' sociodemographic and clinical characteristics with the likelihood of being adherent/mostly adherent.

\section{Statistical analysis}

Descriptive analyses comparing demographic, tumour-related and treatment-related data for CRC survivors according to overall adherence status (adherent/mostly adherent, partially adherent, poorly adherent) with surveillance guidelines will be conducted. Differences in categorical variables between the three surveillance categories will be assessed by $\chi^{2}$ statistics. Differences in continuous variables will be assessed by analysis of variance or appropriate non-parametric techniques.

Binomial regression will be used to obtain absolute and relative measures for the likelihood of being adherent/ mostly adherent according to patients' characteristics. Analytical models will be run separately based on tumour site (colon/rectum) and uniformity of surveillance recommendations (eg, stages I, II/III). Based on our past research on receipt of CRC treatment, ${ }^{4041467}$ the association of sociodemographic and clinical variables including age at diagnosis, year of diagnosis, race/ethnicity, gender, marital status, urban-rural status, census tract poverty level, dual Medicare/Medicaid eligibility, disease stage (stage III vs stage II), tumour grade and tumour location (proximal vs distal) with adherence to surveillance testing guidelines will be evaluated.

We will use survival models (eg, Cox, parametric) to obtain HRs for the relative risk of cancer-specific and all-cause mortality according to the three categories of surveillance. We will also explore the relationship between adherence to specific surveillance tests and survival. All survival models will be explored to present the most clear information regarding the relationship between surveillance and both CSS and OS. In conducting the described 
survival analyses, we will use both propensity score (adherent/mostly adherent vs poorly/non-adherent) and multivariable methods to assess the impact of potential confounders on effect estimates for surveillance guidelines. As noted above for the binomial models, statistical adjustment for the potential confounding effect of the aforementioned sociodemographic and clinical variables will be accounted for in statistical models.

CRC cases with missing values for relevant independent variables will be coded as missing. We will conduct a sensitivity analysis whereby we compare results for our primary analysis where cases with missing values are excluded versus retained in survival models.

\section{Appropriate comparisons for the assessment of surveillance with survival}

For the survival analyses described above, it is necessary to construct similar comparison groups to minimise the potential for biased estimates. In constructing the appropriate comparison groups, as was described for the aforementioned binomial models, separate survival models will be analysed according to tumour typography (colon/ rectum), surveillance guidelines and treatment received. For example, as the surveillance guidelines are the same, we will combine patients with colon cancer with stages II/III disease who received both surgery and adjuvant chemotherapy as treatment. This will ensure that the time from diagnosis to study baseline (1 year following treatment completion) will be approximately the same given the requirements for receiving treatment within specified time periods as described earlier. In this model, adjusted for potential confounders, disease stage would also be a predictive and possibly a modifying variable for the impact of adherence to surveillance guidelines on survival. Stage I patients will be analysed separately as they are only required to receive colonoscopy to be adherent with guidelines.

\section{CONCLUSION}

This study will produce new knowledge for a highly important clinical question-does CRC surveillance testing improve both CSS and OS for patients in the post-treatment phase of care? If our hypothesis is correct (greater adherence to surveillance guidelines improves both CSS and OS), this will inform the practice of evidence-based medicine and help ensure that patients with CRC are receiving proper cancer surveillance testing. This change in practice will result in improved CRC survivorship care, elimination of disparities due to variation in practice, better quality/quantity of life and improved clinical outcomes on a population level to continue the declining mortality rate associated with CRC.

Contributors RBH is responsible for all aspects of the study including conceiving of and designing the study, acquiring the data, analysing and interpreting the data, and drafting and submitting manuscripts. MJHJ and KC are responsible for data cleaning, management and analysis. VL and AS contributed to the study design. ST assisted with the conduct of the study. SZ contributed to the study's design and analysis. All authors critically revised the manuscript and approved of the final version.

Funding To conduct this research project, $\mathrm{RBH}$ received research awards from the University Central of Florida College of Medicine (UCF COM) and the UCF COM Department of Internal Medicine.

Competing interests None declared.

Patient consent Not required.

Ethics approval This study was approved by the National Cancer Institute and the Institutional Review Board of the University of Central Florida.

Provenance and peer review Not commissioned; externally peer reviewed.

Open Access This is an Open Access article distributed in accordance with the Creative Commons Attribution Non Commercial (CC BY-NC 4.0) license, which permits others to distribute, remix, adapt, build upon this work non-commercially, and license their derivative works on different terms, provided the original work is properly cited and the use is non-commercial. See: http://creativecommons.org/ licenses/by-nc/4.0/

(c) Article author(s) (or their employer(s) unless otherwise stated in the text of the article) 2018. All rights reserved. No commercial use is permitted unless otherwise expressly granted.

\section{REFERENCES}

1. American Cancer Society. Cancer Treatment \& Survivorship Facts \& Figures 2016-2017. Atlanta: American Cancer Society, 2016.

2. American Cancer Society. Colorectal Cancer Facts \& Figures 201416. Atlanta: American Cancer Society, 2014.

3. Devesa JM, Morales V, Enriquez JM, et al. Colorectal cancer. The bases for a comprehensive follow-up. Dis Colon Rectum 1988;31:636-52.

4. Kjeldsen BJ, Kronborg O, Fenger C, et al. The pattern of recurrent colorectal cancer in a prospective randomised study and the characteristics of diagnostic tests. Int $\mathrm{J}$ Colorectal Dis 1997;12:329-34.

5. Renouf DJ, Woods R, Speers C, et al. Improvements in 5-year outcomes of stage II/III rectal cancer relative to colon cancer. Am J Clin Oncol 2013;36:558-64.

6. Kievit J. Follow-up of patients with colorectal cancer: numbers needed to test and treat. Eur J Cancer 2002;38:986-99.

7. National Comprehensive Cancer Network. NCCN Clinical Practice Guidelines in Oncology: Colon Cancer. http://www.nccn.org/ professionals/physician_gls/pdf/colon.pdf

8. National Comprehensive Cancer Network. NCCN Clinical Practice Guidelines in Oncology: Rectal Cancer. http://www.nccn.org/ professionals/physician_gls/pdf/rectal.pdf

9. Earle C, Annis R, Sussman J, et al. Follow-up care, surveillance protocol, and secondary prevention measures for survivors of colorectal cancer. Toronto (ON): Cancer Care Ontario, 2012. Program in Evidence-based Care Evidence-Based Series No.: 262.

10. Meyerhardt JA, Mangu PB, Flynn PJ, et al. Follow-up care, surveillance protocol, and secondary prevention measures for survivors of colorectal cancer: American Society of Clinical Oncology clinical practice guideline endorsement. J Clin Oncol 2013;31:4465-70.

11. Cooper GS, Kou TD, Reynolds HL. Receipt of guidelinerecommended follow-up in older colorectal cancer survivors : a population-based analysis. Cancer 2008;113:2029-37.

12. Salloum RG, Hornbrook MC, Fishman PA, et al. Adherence to surveillance care guidelines after breast and colorectal cancer treatment with curative intent. Cancer 2012;118:5644-51.

13. Salz T, Weinberger M, Ayanian JZ, et al. Variation in use of surveillance colonoscopy among colorectal cancer survivors in the United States. BMC Health Serv Res 2010;10:256.

14. Young PE, Womeldorph CM, Johnson EK, et al. Early detection of colorectal cancer recurrence in patients undergoing surgery with curative intent: current status and challenges. $J$ Cancer 2014;5:262-71.

15. Primrose JN, Perera R, Gray A, et al. Effect of 3 to 5 years of scheduled CEA and CT follow-up to detect recurrence of colorectal cancer: the FACS randomized clinical trial. JAMA 2014;311:263-70.

16. Jeffery M, Hickey BE, Hider PN. Follow-up strategies for patients treated for non-metastatic colorectal cancer. Cochrane Database Syst Rev 2007;1:CD002200. 
17. Ohlsson B, Breland U, Ekberg $\mathrm{H}$, et al. Follow-up after curative surgery for colorectal carcinoma. Randomized comparison with no follow-up. Dis Colon Rectum 1995;38:619-26.

18. Tjandra JJ, Chan MK. Follow-up after curative resection of colorectal cancer: a meta-analysis. Dis Colon Rectum 2007;50:1783-99.

19. Jeffery M, Hickey BE, Hider PN, et al. Follow-up strategies for patients treated for non-metastatic colorectal cancer. Cochrane Database Syst Rev 2016;11:CD002200.

20. Mokhles S, Macbeth F, Farewell V, et al. Meta-analysis of colorectal cancer follow-up after potentially curative resection. $\mathrm{Br} J$ Surg 2016;103:1259-68.

21. Wang T, Cui Y, Huang WS, et al. The role of postoperative colonoscopic surveillance after radical surgery for colorectal cancer: a prospective, randomized clinical study. Gastrointest Endosc 2009;69(3 Pt 2):609-15.

22. Rosati G, Ambrosini G, Barni S, et al. A randomized trial of intensive versus minimal surveillance of patients with resected Dukes B2-C colorectal carcinoma. Ann Oncol 2016;27:274-80.

23. Hines RB, Al-Rajabi R. Reply to the letter to the editor 'A randomized trial of intensive versus minimal surveillance of patients with resected Dukes B2-C colorectal carcinoma' by Rosati et al. Ann Oncol 2016;27:957-8

24. Strand E, Nygren I, Bergkvist L, et al. Nurse or surgeon followup after rectal cancer: a randomized trial. Colorectal Dis 2011;13:999-1003.

25. Augestad KM, Norum J, Dehof S, et al. Cost-effectiveness and quality of life in surgeon versus general practitioner-organised colon cancer surveillance: a randomised controlled trial. BMJ Open 2013;3:e002391.

26. Hansdotter Andersson P, Wille-Jørgensen P, Horváth-Puhó E, et al. The COLOFOL trial: study design and comparison of the study population with the source cancer population. Clin Epidemiol 2016:8:15-21.

27. Lyman GH, Levine M. Comparative effectiveness research in oncology: an overview. J Clin Oncol 2012;30:4181-4.

28. Hershman DL, Wright JD. Comparative effectiveness research in oncology methodology: observational data. J Clin Oncol 2012;30:4215-22.

29. World Health Organization. International Agency for Research on Cancer- International Classification of Disease for Oncology. 2016 http://codes.iarc.fr/topography (accessed 12 Jul 2017).

30. Lévesque LE, Hanley JA, Kezouh A, et al. Problem of immortal time bias in cohort studies: example using statins for preventing progression of diabetes. BMJ 2010;340:b5087.

31. Hu CY, Delclos GL, Chan W, et al. Post-treatment surveillance in a large cohort of patients with colon cancer. Am J Manag Care 2011;17:329-36.

32. Brawarsky P, Neville BA, Fitzmaurice GM, et al. Surveillance after resection for colorectal cancer. Cancer 2013;119:1235-42.
33. Mollica MA, Enewold LR, Lines LM, et al. Examining colorectal cancer survivors' surveillance patterns and experiences of care: a SEER-CAHPS study. Cancer Causes Control 2017;28:1133-41.

34. Hawkins NA, Berkowitz Z, Rodriguez J, et al. Examining adherence with recommendations for follow-up in the prevention among colorectal cancer survivors study. Oncol Nurs Forum 2015:42:233-40.

35. Tan AS, Moldovan-Johnson M, Parvanta S, et al. Patient-clinician information engagement improves adherence to colorectal cancer surveillance after curative treatment: results from a longitudinal study. Oncologist 2012;17:1155-62.

36. Amin MB, Edge SB, et al. AJCC cancer staging manual. 8th Edn. Switzerland: Springer, 2017.

37. Sanoff HK, Carpenter WR, Stürmer T, et al. Effect of adjuvant chemotherapy on survival of patients with stage III colon cancer diagnosed after age 75 years. J Clin Oncol 2012;30:2624-34.

38. Sanoff HK, Carpenter WR, Martin CF, et al. Comparative effectiveness of oxaliplatin vs non-oxaliplatin-containing adjuvant chemotherapy for stage III colon cancer. J Natl Cancer Inst 2012;104:211-27.

39. Mihmanlı M, Kabul Gürbulak E, Akgün İE, et al. Delaying surgery after neoadjuvant chemoradiotherapy improves prognosis of rectal cancer. World J Gastrointest Oncol 2016;8:695-706.

40. Hines RB, Barrett A, Twumasi-Ankrah P, et al. Predictors of guideline treatment nonadherence and the impact on survival in patients with colorectal cancer. J Natl Compr Canc Netw 2015;13:51-60.

41. Hines RB, Bimali M, Johnson AM, et al. Prevalence and survival benefit of adjuvant chemotherapy in stage III colon cancer patients: Comparison of overall and age-stratified results by multivariable modeling and propensity score methodology in a population-based cohort. Cancer Epidemiol 2016:44:77-83.

42. National Cancer Institute. Division of Cancer Control \& Population Sciences. SEER-Medicare: SEER Program \& Data. 2017. http:// healthcaredelivery.cancer.gov/seermedicare/aboutdata/program.htm

43. National Cancer Institute. Division of Cancer Control \& Population Sciences. NCl Comorbidity Index Overview. 2017. https:// healthcaredelivery.cancer.gov/seermedicare/considerations/ comorbidity.html

44. Rane PB, Madhavan SS, Sambamoorthi U, et al. Treatment and survival of medicare beneficiaries with colorectal cancer: a comparative analysis between a rural state cancer Registry and National Data. Popul Health Manag 2017;20:55-65.

45. Noone AM, Lund JL, Mariotto A, et al. Comparison of SEER Treatment Data With Medicare Claims. Med Care 2016;54:e55-64.

46. Hines R, Markossian T, Johnson A, et al. Geographic residency status and census tract socioeconomic status as determinants of colorectal cancer outcomes. Am J Public Health 2014;104:e63-71.

47. Hines RB, Markossian TW. Differences in late-stage diagnosis, treatment, and colorectal cancer-related death between rural and urban African Americans and whites in Georgia. $J$ Rural Health 2012;28:296-305. 